\title{
Risco de fratura osteoporótica em portadores de doença pulmonar obstrutiva crônica
}

\author{
Assessment of osteoporotic fracture risk in patients with chronic obstructive pulmonary disease \\ Fractura osteoporótica en enfermedad pulmonar obstructiva crónica
}

Recebido: 08/04/2021 | Revisado: 14/04/2021 | Aceito: 17/04/2021 | Publicado: 27/04/2021

\author{
Daniel Silva Santos \\ ORCID: https://orcid.org/0000-0003-1496-9333 \\ Universidade Tiradentes, Brasil \\ E-mail: danielsiltos@gmail.com \\ Francisco de Assis Pereira \\ ORCID: https://orcid.org/0000-0002-8464-6188 \\ Universidade Federal de Sergipe, Brasil \\ E-mail: frasp@ terra.com.br \\ Janicelma Santos Lins \\ ORCID: https://orcid.org/0000-0002-0370-9723 \\ Universidade Tiradentes, Brasil \\ E-mail: janicelmalins@ig.com.br \\ Isabelle Araujo de Oliveira Santana \\ ORCID: https://orcid.org/0000-0001-8493-9864 \\ Universidade Tiradentes, Brasil \\ E-mail: isabellearaujoos@gmail.com \\ Maria Nathália Prado Simões Mendonça \\ ORCID: https://orcid.org/0000-0001-8529-7883 \\ Universidade Tiradentes, Brasil \\ E-mail: nathaliaprado23@gmail.com \\ Bernard Pereira Reis Barbosa \\ ORCID: https://orcid.org/0000-0003-1757-2358 \\ Universidade Tiradentes, Brasil \\ E-mail: bernard.reis.barbosa@gmail.com \\ Isadora Cardozo Roza Barreto \\ ORCID: https://orcid.org/0000-0001-5317-3309 \\ Universidade Tiradentes, Brasil \\ E-mail: isadora.roza@ souunit.com.br \\ José Joaquim dos Santos Neto \\ ORCID: https://orcid.org/0000-0002-9183-4427 \\ Universidade Tiradentes, Brasil \\ E-mail: jose.jdos@souunit.com.br \\ Igor Sandes de Barcelos \\ ORCID: https://orcid.org/0000-0001-8202-840X \\ Universidade Tiradentes, Brasil \\ E-mail: tegosan@gmail.com
}

\begin{abstract}
Resumo
Objetivo: Avaliar o risco de fratura osteoporótica e a indicação de tratamento para osteoporose em pacientes com Doença Pulmonar Obstrutiva Crônica (DPOC) através do FRAX e NOGG. Métodos: Estudo descritivo, observacional e transversal com amostra de conveniência realizado em um hospital beneficente da cidade de Aracaju-SE. Foram selecionados para o estudo os portadores da DPOC e pareados com indivíduos saudáveis. Resultados: A casuística do estudo foi composta por 174 indivíduos, sendo 87 no grupo DPOC (GD) e 87 no grupo controle (GC). A partir do FRAX clínico para risco absoluto de fratura osteoporótica maior para todos os voluntários do estudo, 3 (3,44\%) do GD possuíram valor indicativo de tratamento para osteoporose e nenhum participante do GC. Para fratura de quadril, foi encontrado que $36(41,4 \%)$ do GD e $12(13,8 \%)$ voluntários do GC teriam indicação de tratamento. Por meio da metodologia NOGG, $44(50,6 \%)$ dos pacientes do GD e $4(4,6 \%)$ dos voluntários do GC tiveram indicação de tratamento, $38(43,7 \%)$ do GD e $58(66,6 \%)$ do GC tiveram indicação de realizar a densitometria óssea. Conclusões: Os dados demonstraram que a DPOC aumenta o risco para fraturas osteoporóticas, principalmente em mulheres e idosos. Por meio do NOGG foi possível identificar o risco de fratura com maior valor preditivo positivo e negativo quando comparado ao FRAX.
\end{abstract}

Palavras-chave: DPOC; Osteoporose; Fraturas por Osteoporose.

\begin{abstract}
Objective: To evaluate the risk of osteoporotic fracture and the indication of treatment for osteoporosis in patients with Chronic Obstructive Pulmonary Disease (COPD) through FRAX and NOGG. Methods: Descriptive, observational and cross-sectional study with a convenience sample carried out in a charity hospital in the city of Aracaju-SE. COPD patients were selected for the study and paired with healthy individuals. Results: The study sample consisted of 174
\end{abstract}


individuals, 87 in the COPD group (DG) and 87 in the control group (CG). Through clinical FRAX for absolute risk of major osteoporotic fracture for all study volunteers, $3(3.44 \%)$ of the DG had indicative treatment value for osteoporosis and no participant in the CG. For hip fractures, it was found that $36(41.4 \%)$ in the GD and 12 (13.8\%) volunteers in the CG would have an indication for treatment. Through the NOGG methodology, $44(50.6 \%)$ of the patients in the GD and $4(4.6 \%)$ of the volunteers in the CG had an indication for treatment, 38 (43.7\%) of the GD and $58(66.6 \%)$ ) of the $\mathrm{CG}$ had an indication to perform bone densitometry. Conclusions: The data demonstrated that COPD increases the risk for osteoporotic fractures, especially in women and the elderly. Through NOGG it was possible to identify the risk of fracture with a higher positive and negative predictive value when compared to FRAX.

Keywords: COPD; Osteoporosis; Osteoporotic Fractures.

\section{Resumen}

Objetivo: Evaluar el riesgo de fractura osteoporótica y la indicación de tratamiento de la osteoporosis en pacientes con Enfermedad Pulmonar Obstructiva Crónica (EPOC) mediante FRAX y NOGG. Métodos: Estudio descriptivo, observacional y transversal con muestra de conveniencia realizado en un hospital benéfico de la ciudad de Aracaju-SE. Los pacientes con EPOC fueron seleccionados para el estudio y emparejados con individuos sanos. Resultados: La muestra de estudio estuvo formada por 174 individuos, 87 en el grupo EPOC (DG) y 87 en el grupo control (GC). Del FRAX clínico para el riesgo absoluto de fractura osteoporótica mayor para todos los voluntarios del estudio, 3 (3,44\%) de la DG tenían un valor de tratamiento indicativo para la osteoporosis y ningún participante en la GC. Para las fracturas de cadera, se encontró que $36(41,4 \%)$ de los GD y $12(13,8 \%)$ voluntarios del GC tendrían indicación de tratamiento. Mediante la metodología NOGG, 44 (50,6\%) de los pacientes del GD y 4 (4,6\%) de los voluntarios del GC tenían indicación de tratamiento, 38 (43,7\%) del GD y $58(66,6 \%)$ ) del GC tenía indicación para realizar densitometría ósea. Conclusiones: Los datos demostraron que la EPOC aumenta el riesgo de fracturas osteoporóticas, especialmente en mujeres y ancianos. A través de NOGG fue posible identificar el riesgo de fractura con un valor predictivo positivo y negativo mayor en comparación con FRAX.

Palabras clave: EPOC; Osteoporosis; Fracturas Osteoporóticas.

\section{Introdução}

A doença pulmonar obstrutiva crônica (DPOC) é uma condição respiratória comum, evitável e tratável. (GOLD, 2020; Han et al., 2015). Apesar de comprometer os pulmões, a progressão da doença está associada a comorbidades significativas, que além de comprometer a qualidade de vida do indivíduo, aumenta o risco de hospitalização e morte. (Schwab et al., 2017). A DPOC está associada a um risco aumentado de câncer de pulmão e doenças cardiovasculares (Rothnie et al., 2018). Além disso, pacientes com DPOC têm alta prevalência de osteoporose ou baixa densidade mineral óssea (DMO) (Lin et al., 2018), considerada a maior comorbidade relacionada a DPOC (Okazaki et al., 2016).

A osteoporose é uma doença esquelética sistêmica, geralmente silenciosa, caracterizada por baixa DMO e/ou deterioração da microarquitetura, que aumenta a fragilidade óssea e por consequência, o risco de fraturas osteoporóticas. (Lewiecki, 2021; Rosen et al., 2018). Historicamente, a avaliação do risco de fratura foi feita apenas por meio da DMO (Kanis et al., 2020). Entretanto, a maioria das fraturas por fragilidade óssea ocorre na faixa não osteoporótica (escore T> - 2,5), enfatizando a influência de outros fatores de risco na incidência de fraturas (Gudmundsson et al., 2019). Assim, em 2008 a Organização Mundial de Saúde (OMS) criou a ferramenta FRAX - Fracture Risk Assessment Tool - uma calculadora computadorizada que estima a probabilidade de fratura de quadril e fraturas maiores (coluna, úmero proximal ou antebraço) em 10 anos para pacientes não tratados com idades entre 40 e 90 anos (Rosen et al., 2018). Para isso, utiliza fatores de risco clínicos para osteoporose, como idade, sexo, índice de massa corporal (IMC) ou DMO, fratura osteoporótica tabagismo atual, uso de glicocorticoides e presença de distúrbios que causam osteoporose secundária (Okazaki et al., 2016). O objetivo dessa ferramenta é identificar indivíduos que se encontrem em alto risco para fraturas osteoporóticas e indicar o tratamento adequado antes que desenvolvam a doença (Pegoraro et al., 2017).

O NOGG (National Osteoporosis Guideline Group), grupo multidisciplinar de especialistas em osteoporose responsável por atualizar as diretrizes, criou, em 2017, uma extensão do FRAX, com o intuito de selecionar os pacientes que necessitam de uma avaliação da DMO e fornecer limiares de intervenção para o tratamento farmacológico (Compston et al., 2017). A ferramenta utiliza as mesmas variáveis da ferramenta FRAX para seu algoritmo além dos valores de risco para fratura 
de quadril e fraturas maiores obtidos através do FRAX, e assim categorizar o risco de fratura osteoporótica em baixo, intermediário e alto risco de fratura e a intervenção adequada para cada uma das categorias (Compston et al., 2017).

As ferramentas de avaliação de risco de fratura ajudam a identificar indivíduos que precisam de tratamento, mas sua utilidade depende da capacidade do clínico de traduzir corretamente seus resultados em prática clínica (Gudmundsson et al., 2019). O objetivo desse estudo foi avaliar o risco para fratura osteoporótica e a indicação de tratamento para osteoporose em pacientes com DPOC através do NOGG.

\section{Metodologia}

Trata-se de um estudo descritivo, quantitativo, observacional e transversal com amostra de conveniência realizado em um hospital beneficente da cidade de Aracaju-SE. O estudo foi composto por dois grupos: o grupo DPOC (GD), com todos os pacientes portadores de DPOC em seguimento no ambulatório de pneumologia e um grupo controle (GC), com indivíduos sem patologias e/ou doenças relacionadas à osteoporose e que não faziam uso de medicações que interferissem no metabolismo ósseo. Os grupos foram pareados para idade, sexo, peso, altura e IMC.

A partir dos dados coletados por meio de questionário, foi realizada a análise do risco de fratura por meio da ferramenta FRAX clínico, referente a população brasileira. Posteriormente, foi aplicada ao NOGG que categoriza os resultados do FRAX em alto risco, risco intermediário e baixo risco.

Para análise estatística foi utilizado o programa GraphPed versão 8.0, 2016 (San Diego, CA, USA). Os resultados foram apresentados como média e desvio-padrão. A avaliação das variáveis numéricas entre dois grupos foi realizada através do teste $t$-Studente Foi considerado um nível de significância de 0,05 .

O estudo foi aprovado pelo Comitê de Ética em Pesquisa com seres humanos da Universidade Tiradentes, com o CAAE: 12804519.3 .0000 .5371$.

\section{Resultados e Discussão}

No estudo foram entrevistados 174 indivíduos, os quais foram divididos em dois grupos. O Grupo Doença (GD) formado por pacientes com DPOC - composto por 87 pacientes portadores de DPOC em seguimento no ambulatório de pneumologia e o grupo controle (GC) foi formado por 87 acompanhantes dos pacientes com DPOC.

Entre os 87 pacientes do GD, 44 (50,6\%) foram do sexo masculino e a média de idade foi de 66,2 anos, comparado com 87 voluntários do GC em que 42 (51,7\%) foram do sexo masculino com a média de idade de 65,5 anos. Em relação às médias dos dados antropométricos encontramos no $\mathrm{GD}$ ( $\mathrm{IMC}=26,6 \mathrm{~kg} / \mathrm{m}^{2}$, peso $=68,9 \mathrm{~kg}$, altura $=1,60 \mathrm{~m}$ ) e no GC ( IMC $=26,6 \mathrm{~kg} / \mathrm{m}^{2}$, peso $=71,3 \mathrm{~kg}$, altura $=1,63 \mathrm{~m}$ ).

Ao serem questionados acerca dos hábitos de vida, foi observado que no GD quatro $(4,6 \%)$ pacientes eram etilistas, $14(16,1 \%)$ tabagistas e $74(85 \%)$ relataram história de tabagismo comparado com sete (8\%) etilistas, 14 (16,1\%) tabagistas e $14(16,1 \%)$ com história de tabagismo no GC. O uso de corticoide foi evidenciado somente no GD, em que $70(80,4 \%)$.

Os episódios de fratura foram mais frequentes no GD, 27 (31\%), dessas, 25 (92,6\%) foram traumáticas, ao passo que oito $(9,2 \%)$ no GC. Em contraponto, a história familiar de fratura de quadril foi menos frequente no GD, em que duas $(2,3 \%)$ pessoas relataram e sete (8\%) no GC.

Ao avaliar o evento de quedas, foi evidenciado que no GD 42 (48,3\%) tem histórico de queda da própria altura nos últimos 12 meses, desses 17 (40,5\%) ocorreram em ambiente domiciliar, 22 (52,4\%) em ambiente não domiciliar e $3(7,1 \%)$ em ambos os ambientes. Já no GC, 17 (19,5\%) relataram episódios de queda.

A presença de outras comorbidades podem ser observados na Tabela 1. 
Tabela 1 - Comorbidades presentes nos participantes do grupo doença (GD) e grupo controle (GC), entrevistados entre outubro de 2019 a março de 2020.

\begin{tabular}{lll}
\hline & GD N(87) / \% & GC N(87) / \% \\
\hline História de tabagismo & $74 / 85,0$ & $14 / 16,1$ \\
\hline Osteoporose & $9 / 10,3$ & $13 / 14,9$ \\
\hline Diabetes Mellitus tipo 2 & $25 / 28,7$ & $36 / 41,4$ \\
\hline Hipertensão arterial & $58 / 66,7$ & $6 / 6,9$ \\
\hline Cardiopatia & $33 / 37,9$ & $6 / 6,9$ \\
\hline Neuropatia & $12 / 13,8$ & 0 \\
\hline Incontinência urinária & $9 / 10,3$ & $1 / 1,1$ \\
\hline AVE & $6 / 6,9$ & $15 / 17,2$ \\
\hline Problemas com o sono & $32 / 36,8$ & $2 / 2,3$ \\
\hline Tireoidopatias & $5 / 5,7$ & $3 / 3,4$ \\
\hline Depressão & $15 / 17,2$ & $9 / 10,3$ \\
\hline Ansiedade & $23 / 26,4$ & $2 / 2,3$ \\
\hline Tontura / vertigem & $28 / 32,2$ & $-1,3$ \\
\hline
\end{tabular}

Fonte: Autores.

Ao calcular o risco absoluto de fraturas em 10 anos sem inclusão de densitometria óssea para a população com DPOC, foi observado que o valor do FRAX clínico para os portadores de DPOC variaram de 1,1 a 26\%, com uma média de 7,2\% de risco para fraturas osteoporóticas maior. No GC, esse risco foi de 3,98 \%. Entre os homens o risco médio de fraturas maiores foi de $5,75 \%$ e entre as mulheres de $8,63 \%$ para o GD, comparado com 2,98\% para homens e 4,93\% para as mulheres do GC.

No que concerne ao risco de fratura de quadril, os valores do GD variaram entre 0,1 e 17 e a média encontrada foi de 3,29\%, já no GC esse risco variou de 0,1 a 7,5\%, com média de 1,45\%. O resultado do risco de fratura de quadril por sexo evidenciou um valor de $2,9 \%$ para o sexo masculino e $3,59 \%$ para o sexo feminino no GD, enquanto que $1,29 \%$ e $1,60 \%$ para os homens e mulheres do GC respectivamente.

Quando avaliado o risco de fratura osteoporótica por faixa etária foi observado aumento do risco de fratura em 10 anos nas faixas etárias maiores em ambos os grupos. O risco de fratura osteoporótica maior e de quadril em 10 anos por idade e sexo encontra-se na Tabela 2. 
Tabela 2 - Médias de risco de fraturas maiores e de quadril calculado pela ferramenta FRAX, segundo sexo e faixa etária nos participantes do grupo doença (GD) e grupo controle (GC), entrevistados entre outubro de 2019 a março de 2020.

\begin{tabular}{|c|c|c|}
\hline & $\begin{array}{c}\text { Risco para fraturas osteoporóticas maior em } 10 \\
\qquad \text { anos } \\
\text { (media } \pm \text { desvio padrão) }\end{array}$ & $\begin{array}{l}\text { Risco para fratura de quadril em } \\
\qquad 10 \text { anos } \\
\text { (media } \pm \text { desvio padrão) }\end{array}$ \\
\hline Todos & $7,2 \% \pm 4,93(\mathrm{GD})$ & $3,29 \% \pm 3,44(\mathrm{GD})$ \\
\hline \multirow[t]{2}{*}{ os pacientes } & $3,98 \% \pm 2,46(\mathrm{GC})$ & $1,45 \% \pm 1,45(\mathrm{GC})$ \\
\hline & $\mathrm{P}=<0,01$ & $\mathrm{P}=<0,01$ \\
\hline \multirow[t]{3}{*}{ Homens } & $5,75 \% \pm 3,40(\mathrm{GD})$ & $2,9 \% \pm 2,75(\mathrm{GD})$ \\
\hline & $2,98 \% \pm 1,35(\mathrm{GC})$ & $1,29 \% \pm 1,13(\mathrm{GC})$ \\
\hline & $\mathrm{P}=<0,01$ & $P=0,0006$ \\
\hline \multirow[t]{3}{*}{ Mulheres } & $8,63 \% \pm 5,76(\mathrm{GD})$ & $3,58 \% \pm 4,01(\mathrm{GD})$ \\
\hline & $4,93 \% \pm 2,87(\mathrm{GC})$ & $1,60 \% \pm 1,70(\mathrm{GC})$ \\
\hline & $P=0,0003$ & $\mathrm{P}=0,0036$ \\
\hline \multirow[t]{3}{*}{40 a 59 anos } & $4,0 \% \pm 2,56(\mathrm{GD})$ & $0,56 \% \pm 0,58(\mathrm{GD})$ \\
\hline & $2,34 \% \pm 1,06(\mathrm{GC})$ & $0,22 \% \pm 0,15(\mathrm{GC})$ \\
\hline & $\mathrm{P}=0,009$ & $\mathrm{P}=0,013$ \\
\hline \multirow[t]{3}{*}{60 a 69 anos } & $6,33 \% \pm 3,39(\mathrm{GD})$ & $2,33 \% \pm 1,84(\mathrm{GD})$ \\
\hline & $3,66 \% \pm 2,05(\mathrm{GC})$ & $1,0 \% \pm 0,59(\mathrm{GC})$ \\
\hline & $P=0,0004$ & $P=0,0003$ \\
\hline \multirow[t]{3}{*}{ Acima de 70 anos } & $9,87 \% \pm 5,78(\mathrm{GD})$ & $5,77 \% \pm 3,88(\mathrm{GD})$ \\
\hline & $5,35 \% \pm 1,96(\mathrm{GC})$ & $2,66 \% \pm 1,62(\mathrm{GC})$ \\
\hline & $\mathrm{P}=<0,01$ & $\mathrm{P}=<0,01$ \\
\hline
\end{tabular}

Fonte: Autores.

Ao considerar os resultados obtidos a partir do FRAX clínico para risco absoluto de fratura osteoporótica maior para todos os voluntários do estudo, apenas $3(3,44 \%)$ do GD possuíram risco absoluto para fraturas de ossos maiores e nenhum participante do GC, isto é, valor do FRAX igual ou maior a 20\%, a partir do qual há indicação para intervenção medicamentosa. Para fratura de quadril, no entanto, o valor do FRAX que indica tratamento é maior ou igual a 3\%, assim foi encontrado que $36(41,4 \%)$ do GD e $12(13,8 \%)$ voluntários do GC teriam indicação de tratamento.

Ao avaliar a indicação de tratamento utilizando o NOGG, foi observado que $44(50,6 \%)$ dos pacientes do GD e 4 $(4,6 \%)$ dos voluntários do GC tiveram indicação de tratamento, $38(43,7 \%)$ do GD e 58 (66,6\%) do GC tiveram indicação de realizar a densitometria óssea. No entanto, de todos os participantes, apenas 9 (10,3\%) do GD e 29 (33,3\%) do GC já tinham sido submetidos a densitometria óssea. As indicações de tratamento de acordo com o FRAX e NOGG estão dispostos na Tabela 3 e as condutas sugeridas pelo NOGG por sexo estão descritas na Tabela 4. 
Tabela 3 - Indicação de tratamento conforme valor do FRAX clínico e NOGG nos participantes do grupo doença (GD) e grupo controle (GC), entrevistados entre outubro de 2019 a março de 2020.

\begin{tabular}{lccc}
\hline & GD $(\mathbf{N}=\mathbf{8 7})$ & GC $(\mathbf{N}=\mathbf{8 7})$ & $\mathbf{P}$ \\
\hline Fratura maior & 3 & 0 & 0,05 \\
\hline FRAX clinico $(>20)$ & 23 & 1 & 0,001 \\
NOGG (alto risco) & & 12 & 0,05 \\
\hline Fratura de quadril & 36 & 3 & 0,001 \\
\hline FRAX clínico (> 3) & 44 & & \\
\hline NOGG (alto risco) & & 3 & \\
\hline
\end{tabular}

Fonte: Autores.

Tabela 4 - Condutas indicadas pelo NOGG pareados por sexo nos participantes do grupo doença (GD) e grupo controle (GC), entrevistados entre outubro de 2019 a março de 2020.

\begin{tabular}{|c|c|c|c|}
\hline & $\begin{array}{c}\text { Observação clínica (n / } \\
\%)\end{array}$ & $\begin{array}{c}\text { Indicação de DMO } \\
\qquad(n / \%)\end{array}$ & $\begin{array}{c}\text { Indicação de tratamento (n / } \\
\qquad)\end{array}$ \\
\hline GD $(n=87)$ & $5 / 5,7 \%$ & $38 / 43,7 \%$ & 44 / 50,6\% \\
\hline Homens $(n=44)$ & $5 / 11,4 \%$ & $18 / 40,9 \%$ & $21 / 47,7 \%$ \\
\hline Mulheres $(n=43)$ & - & $20 / 46,5 \%$ & $23 / 53,5 \%$ \\
\hline GC $(n=87)$ & $25 / 28,7 \%$ & $58 / 66,7 \%$ & $4 / 4,6 \%$ \\
\hline Homens $(\mathrm{n}=42)$ & $13 / 30,9 \%$ & $27 / 64,3 \%$ & $2 / 4,8 \%$ \\
\hline Mulheres $(\mathrm{n}=45)$ & $12 / 26,7 \%$ & $31 / 68,9 \%$ & $2 / 4,4 \%$ \\
\hline
\end{tabular}

Fonte: Autores.

A partir desses resultados, foi feita uma comparação entre as indicações de tratamento pelo FRAX e NOGG, os resultados obtidos encontram-se na Tabela 5.

Tabela 5 - Comparação das indicações de tratamento para osteoporose utilizando o FRAX clínico e o NOGG pareados por sexo para os participantes do GD e do GC entrevistados entre outubro de 2019 a março de 2020.

\begin{tabular}{lcccc}
\hline \multicolumn{1}{c}{ Indicação de tratamento } & $\begin{array}{c}\text { Pelo FRAX } \\
(\mathrm{n} / \%)\end{array}$ & $\begin{array}{c}\text { Pelo NOGG } \\
(\mathrm{n} / \%)\end{array}$ & $\mathbf{P}$ & $\begin{array}{c}\text { Por ambos } \\
(\mathrm{n} / \%)\end{array}$ \\
\hline Grupo Doença $(\mathrm{n}=87)$ & $\mathbf{3 6} / \mathbf{4 1 , 4 \%}$ & $\mathbf{4 4} / \mathbf{5 0 , 6 \%}$ & $\mathbf{0 , 0 1}$ & $\mathbf{3 1} / \mathbf{3 5 , 6 \%}$ \\
Homens $(\mathrm{n}=44)$ & $17 / 38,6 \%$ & $21 / 47,7 \%$ & $\mathbf{0 , 0 5}$ & $14 / 31,8 \%$ \\
Mulheres $(\mathrm{n}=43)$ & $19 / 44,2 \%$ & $23 / 53,5 \%$ & $\mathbf{0 , 0 5}$ & $17 / 39,5 \%$ \\
\hline Grupo Controle $(\mathrm{n}=87)$ & $\mathbf{1 2} / \mathbf{1 3 , 8 \%}$ & $\mathbf{4 / 4 , 6 \%}$ & $\mathbf{0 , 0 1}$ & $\mathbf{2} / \mathbf{2 , 3 \%}$ \\
Homens $(\mathrm{n}=42)$ & $4 / 9,5 \%$ & $2 / 4,8 \%$ & $\mathbf{0 , 0 5}$ & $1 / 2,4 \%$ \\
Mulheres $(\mathrm{n}=45)$ & $8 / 17,7 \%$ & $2 / 4,4 \%$ & $\mathbf{0 , 0 5}$ & $1 / 2,2 \%$ \\
\hline
\end{tabular}

Fonte: Autores. 
Ao comparar o GD com o GC, foi observado que a DPOC está associada ao risco mais elevado para fratura maiores $(\mathrm{p}<0,01)$ bem como de quadril $(\mathrm{p}<0,01)$.

A respeito dos aspectos sociodemográficos da população estudada, 50\% da amostra dos pacientes com DPOC foi composta por homens e a média de idade foi superior a 60 anos. O mesmo foi observado em uma coorte americana realizada com 4477 participantes portadores de DPOC, que relatou predominância sexo masculino (56\%) e média de idade de 63 anos. Esses achados já eram esperados, visto que segundo o GOLD, idade avançada é fator de risco independente tanto para DPOC quanto para o fratura óssea por fragilidade (GOLD, 2020; Park et al., 2021).

No tocante aos fatores de risco para osteoporose, observou-se que tabagismo atual ou prévio foi 4,5 vezes mais frequente na população com DPOC. Ao analisar o estudo de Adas-Okuma, em que uma população de 91 pacientes com DPOC e 81 controles pareados por idade e sexo foram avaliados através da DMO, o grupo DPOC teve 2,7 vezes mais exposição tabágica que o controle, concordando com os resultados encontrados no atual estudo. Embora frequentemente associado ao risco para fraturas osteoporóticas, o tabagismo ainda é ponto de divergências na literatura (Okuma et al., 2020). Nesse âmbito, em um estudo multicêntrico feito com uma população de homens americanos acima dos 65 anos, diagnosticados com asma ou DPOC, o tabagismo foi um dos responsáveis pela perda de DMO e aumento do risco de fratura por fragilidade (Dam et al., 2010). Já outro estudo realizado em 2017 em Joinville, a história pregressa de tabagismo não teve relação com a fratura por fragilidade do quadril (Silva et al., 2017).

Ainda referente aos fatores de risco, ao analisar o uso de glicocorticoides, foi observado uma predominância significativa no GD, e como apontado por Kenneth Saag (2018), essa medicação é fator de risco independente para fratura e seu efeito na massa óssea se assemelha aos efeitos vistos na DPOC, no tabagismo crônico e no baixo IMC (comum no enfisema) (Saag et al., 2018). Portanto, é plausível que a associação destes fatores estejam implicados na fisiopatologia da perda de massa óssea nos portadores da DPOC.

A convergência dos fatores de risco expostos e a natureza inflamatória da DPOC ultimam para o maior risco de fratura evidenciado no GD. Adas-Okuma et al. corroboram esse achado, relatando que para o mesmo T-score, indivíduos com DPOC tinham 4,7 vezes mais chance de fratura que indivíduos saudáveis (Okuma et al., 2020). Dessa forma, a analise somente da DMO é ineficaz em predizer a saúde óssea nessa população e a melhor estratégia para prevenção primária é a identificação, por parte do médico, dos fatores de risco clínicos (Curtis et al., 2020).

Outrossim, é relevante destacar que a oferta de densitometria óssea pelo SUS ainda é insuficiente para o rastreio populacional, uma vez que apenas em 9,6\% dos municípios brasileiros possuem o equipamento necessário para realizar o exame (Arruda et al., 2013; Pereira 2017). Os dados do presente estudo mostram que dos 96 participantes com indicação de realização da densitometria, apenas 38 (39,5\%) haviam realizado o exame previamente, o que pode ser reflexo dessa baixa disponibilidade.

No que tange ao risco de fratura estimado pelo FRAX, esse foi acentuadamente maior no GD em todas faixas etárias e em ambos sexos, com maior risco absoluto em mulheres e idosos. A literatura corrobora a esses achados, visto que no estudo de Adas-Okuma esse mesmo perfil demográfico foi responsável pelo maior número de fraturas da população (Okuma et al., 2020).

Apesar disso, o estudo GLOW (Global Longitudinal Study of Osteoporosis in Women) afirma que uma limitação do FRAX é não englobar algumas comorbidades que alteram consideravelmente a saúde óssea e completa ainda que a inclusão de tais doenças, inclusive DPOC, aumenta a acurácia na detecção de fraturas (Dennison et al., 2012). Por conseguinte, é possível presumir que os valores referentes ao risco de fraturas encontrados no presente estudo estejam subestimados.

Seguindo as recomendações terapêuticas estabelecidas pelo FRAX e NOGG, é importante observar que apesar do atual estudo ter encontrado um número significativo de indicações de tratamento para osteoporose no GD, nenhum participante 
desse grupo estava em uso de medicação específica para a doença. A explicação para tal achado pode estar atrelada ao desconhecimento de muitos profissionais da associação da DPOC com baixa massa óssea, bem como o acesso restrito a DMO e a aplicação das ferramentas clínicas.

Dito isso, embora recente, o NOGG já é usado no Reino Unido por médicos generalistas e especialistas e recomendado por alguns autores no Brasil como ferramenta para selecionar os pacientes que necessitam: de tratamento farmacológico precoce, avaliação da DMO, e mudanças comportamentais a fim de prevenir fraturas osteoporóticas. (Compston et al., 2017; Radominski et al., 2017).

Ao comparar as indicações de tratamento sugeridas pelo FRAX e NOGG, observou-se que o último indicou mais tratamento para o GD e menos para o GC. Isso pode ser reflexo de um maior valor preditivo positivo e negativo para indicar terapêutica pela ferramenta NOGG, entretanto, novos estudos comparativos precisam ser realizados a fim de se chegar a uma conclusão segura. Tais dados apresentados concordam com um estudo realizado no hospital escola da Universidade Federal de Sergipe, em que foi avaliado o risco de fratura osteoporótica em idosos e indicação de tratamento por meio do FRAX e NOGG e detectou que esse foi capaz de detectar indivíduos de risco de forma mais precoce (Silva 2019).

Sobre as limitações do presente estudo, a primeira é que para uma avaliação mais completa da saúde óssea dos entrevistados, a DMO teria grande valia, entretanto, a utilização de tal ferramenta foi inviável na população estudada por motivos financeiros e baixa disponibilidade nos serviços públicos. Além disso, vale ressaltar que pela natureza transversal do estudo, não foi avaliada a incidência de fraturas. Outra limitação encontrada diz respeito a ausência de dados sobre o grau de gravidade da DPOC do GD, o que poderia contribuir no entendimento do efeito deletério da DPOC sobre a massa óssea. Uma última limitação esteve relacionada com o fato de não ter sido avaliado os marcadores bioquímicos de reabsorção óssea, os quais sofrem influencia do uso de glicocorticoides, medicação prevalente em população estudada.

Por fim, a realização do atual estudo trouxe o benefício da avaliação mais cuidadosa e criteriosa dos riscos de fratura na população do serviço avaliado e a perspectiva para o futuro do uso das ferramentas aplicadas serem cada vez mais inseridas na prática da saúde pública.

\section{Conclusão}

O estudo demonstrou que a DPOC tem um impacto significativo sobre a qualidade óssea, tendo em vista que quando comparado o NOGG entre os participantes portadores de DPOC e os saudáveis, foi obtido maior indicação de tratamento para osteoporose no grupo doença.

No tocante aos fatores de risco para osteoporose, o uso de corticoide foi o mais frequente no grupo de pacientes com DPOC, seguido de história de fratura prévia e tabagismo.

Os dados evidenciaram também que o sexo feminino possui maior risco de fratura quando comparado ao sexo masculino, uma vez que o numero de mulheres com categoria do NOGG de alto risco foi maior que a número de homens na mesma categoria. Além disso, quanto maior a idade, maior o risco de fratura osteoporótica calculada pelo NOGG.

Ao comparar indicação de tratamento pelas ferramentas FRAX clínico e NOGG, foi observado que o GD teve maior indicação terapêutica pelo NOGG, já no GC, essa indicação foi maior pelo FRAX.

Diante do exposto, é possível caracterizar as fraturas osteoporóticas como um problema de saúde pública, que deve ser estudado a fim de aprimorar o diagnóstico e tratamento da osteoporose, bem como a prevenção de fraturas por fragilidade.

Novos estudos sobre o uso dessas ferramentas clínicas em pacientes com potencial risco de osteoporose devem ser realizados com o objetivo de tornar mais acurada a estratégia de prevenção de fraturas osteoporóticas e reduzir as consequências advindas desse problema. 


\section{Referências}

Adas-Okuma, M. G., Maeda, S. S., Gazzotti, M. R., Roco, C. M., Pradella, C. O., Nascimento, O. A., ... \& Lazaretti-Castro, M. (2020). COPD as an independent risk factor for osteoporosis and fractures. Osteoporosis International, 31(4), 687-697.

Arruda, M. F., Pereira, L. G. A., Fávero, T. M., Cassettari, L. L., \& de Souza Junior, A. P. (2013). Perfil do Uso do Programa Avaliacional Sapori em Paralelo à Necessidade da Densitometria Mineral Óssea em Amostra de Mulheres Pós-Menopáusicas da Cidade de Catanduva-SP. Saúde e Pesquisa, 6(2).

Compston, J., Cooper, A., Cooper, C., Gittoes, N., Gregson, C., Harvey, N., ... \& Vine, N. (2017). UK clinical guideline for the prevention and treatment of osteoporosis. Archives of osteoporosis, 12(1), 43.

Curtis, E. M., Woolford, S., Holmes, C., Cooper, C., \& Harvey, N. C. (2020). General and Specific Considerations as to why Osteoporosis-Related Care Is Often Suboptimal. Current osteoporosis reports, 18(1), 38-46.

Dam, T. T., Harrison, S., Fink, H. A., Ramsdell, J., \& Barrett-Connor, E. (2010). Bone mineral density and fractures in older men with chronic obstructive pulmonary disease or asthma. Osteoporosis International, 21(8), 1341-1349.

Dennison, E. M., Compston, J. E., Flahive, J., Siris, E. S., Gehlbach, S. H., Adachi, J. D., ... \& Glow Investigators. (2012). Effect of co-morbidities on fracture risk: findings from the Global Longitudinal Study of Osteoporosis in Women (GLOW). Bone, 50(6), 1288-1293.

Global Initiative for Chronic Obstructive Lung Disease - GOLD. https://goldcopd.org/. Accessed April 11, 2019

Gudmundsson, H. T., Hansen, K. E., Halldorsson, B. V., Ludviksson, B. R., \& Gudbjornsson, B. (2019). Clinical decision support system for the management of osteoporosis compared to NOGG guidelines and an osteology specialist: a validation pilot study. BMC medical informatics and decision making, 19(1), 1-8.

Han, M. K., Dransfield, M. T., \& Martinez, F. J. (2018). Chronic obstructive pulmonary disease: Definition, clinical manifestations, diagnosis, and staging. Uptodate. May, 14.

Kanis, J. A., Harvey, N. C., Johansson, H., Liu, E., Vandenput, L., Lorentzon, M., ... \& McCloskey, E. V. (2020). A decade of FRAX: how has it changed the management of osteoporosis?. Aging clinical and experimental research, 32(2), 187-196.

Lewiecki, E. M., \& Schmader, K. E. (2021). Osteoporotic fracture risk assessment. Uptodate. Mar, 09

Lin, C. H., Chen, K. H., Chen, C. M., Chang, C. H., Huang, T. J., \& Lin, C. H. (2018). Risk factors for osteoporosis in male patients with chronic obstructive pulmonary disease in Taiwan. PeerJ, 6, e4232.

Okazaki, R., Watanabe, R., \& Inoue, D. (2016). Osteoporosis associated with chronic obstructive pulmonary disease. Journal of bone metabolism, $23(3), 111$.

Park, H. Y., Lee, H., Kang, D., Choi, H. S., Ryu, Y. H., Jung, K. S., ... \& Yoo, K. H. (2021). Understanding racial differences of COPD patients with an ecological model: two large cohort studies in the US and Korea. Therapeutic advances in chronic disease, 12, 2040622320982455.

Pegoraro, T., de Oliveira Padilha, E. L., Conti, C. F. B., \& da Silva, L. M. (2017). Quantificação do risco de fraturas através da ferramenta FRAX em pacientes com doença renal crônica em hemodiálise. Arquivos Catarinenses de Medicina, 46(3), 39-58.

Pereira, A. S. et al. (2018). Metodologia da pesquisa científica. [e-book]. Santa Maria. Ed. UAB/NTE/UFSM. https://repositorio.ufsm.br/bitstream/handle/1/15824/Lic_Computacao_Metodologia-Pesquisa-Cientifica.pdf?sequence=1 .

Pereira, E. N. L. (2017). Análise da distribuição dos equipamentos de densitometria óssea no Brasil: importância da gestão eficiente de tecnologias em saúde.

Radominski, S. C., Bernardo, W., Paula, A. P. D., Albergaria, B. H., Moreira, C., Fernandes, C. E., ... \& Borba, V. Z. (2017). Diretrizes brasileiras para o diagnóstico e tratamento da osteoporose em mulheres na pós-menopausa. Revista Brasileira de Reumatologia, 57, s452-s466.

Rosen, H. N., Drezner, M. K., Rosen, C. J., Schmader, K. E., \& Mulder, J. E. (2017). Overview of the management of osteoporosis in postmenopausal women. Monografía en Internet]. Uptodate.

Rothnie, K. J., Connell, O., Müllerová, H., Smeeth, L., Pearce, N., Douglas, I., \& Quint, J. K. (2018). Myocardial infarction and ischemic stroke after exacerbations of chronic obstructive pulmonary disease. Annals of the American Thoracic Society, 15(8), 935-946.

Saag, K., \& Adler, R. A. (2018). Glucocorticoid-induced osteoporosis. Primer on the metabolic bone diseases disorder of mineral metabolism. 9th Ed. New Jersey, USA: Wiley, 467-473.

Schwab, P., Dhamane, A. D., Hopson, S. D., Moretz, C., Annavarapu, S., Burslem, K., ... \& Kaila, S. (2017). Impact of comorbid conditions in COPD patients on health care resource utilization and costs in a predominantly Medicare population. International journal of chronic obstructive pulmonary disease, 12,735 .

Silva, D. D. C. (2019). Indicação de tratamento para osteoporose em idosos ambulatoriais do Hospital Universitário da Universidade Federal de Sergipe.

Silva, D. M. W., Borba, V. Z. C., \& Kanis, J. A. (2017). Evaluation of clinical risk factors for osteoporosis and applicability of the FRAX tool in Joinville City, Southern Brazil. Archives of osteoporosis, 12(1), 1-8. 